\title{
Deslocamento ativo em adolescentes: prevalência e preditores associados ao trajeto casa-escola
}

\author{
Active commuting among adolescents: prevalence and associated predictors at the \\ home-school route
}

\section{AUTORES \\ Mabliny Thuany ${ }^{1}$ (D) \\ Fernanda Karina dos $\operatorname{Santos}^{2}$ (D) \\ Marcos Bezerra de Almeida ${ }^{3}$ (D) \\ Thayse Natacha Queiroz Ferreira Gomes ${ }^{3}$ (D) \\ 1 Faculdade de Desporto da Universidade do Porto, Centro de Investigação, Formação, Intervenção e \\ Inovação em Desporto, Porto, Portugal. \\ 2 Universidade Federal de Viçosa, Departamento de Educação Física, Viçosa, Minas Gerais, Brasil. \\ 3 Universidade Federal de Sergipe, Departamento de Educação Física, São Cristóvão, Sergipe, Brasil.}

\section{CONTATO}

Thayse Natacha Queiroz Ferreira Gomes thayse_natacha@hotmail.com

Universidade Federal de Sergipe, Departamento de Educação Física - Cidade Universitária Prof. José Aloísio de Campos, Av. Marechal Rondon, s/n, Jd. Rosa Elze, São Cristóvão, Sergipe, Brasil.

CEP: 49100-000.

DOI

$10.12820 /$ rbafs.26e0203

\section{(cc) BY}

Este trabalho está licenciado com uma Licença Creative Commons - Atribuição 4.0 Internacional.

\begin{abstract}
RESUMO
O objetivo do presente estudo é descrever a prevalência de deslocamento ativo entre adolescentes brasileiros no trajeto casa-escola considerando as unidades federativas e regiões do país, e verificar os preditores associados a esse comportamento. A amostra foi composta por 99.967 adolescentes (ambos os sexos; média de idade 14,24 anos) que participaram da Pesquisa Nacional de Saúde do Escolar em 2015. Para o estudo, foram consideradas informações relativas ao deslocamento ativo no percurso casa-escola (frequência e duração), aspecto socioeconômico e percepção de segurança no trajeto para a escola, sendo obtidas através de questionário. A análise estatística foi realizada por meio da regressão logística binária. Foi observado que $44,4 \%$ dos jovens realizam o deslocamento ativo (ir e voltar da escola através do uso de bicicleta/caminhada, pelo menos cinco dias na semana), sendo que a região Sudeste apresenta a maior porcentagem de adolescentes que aderem à tal prática $(46,4 \%)$. Ser do sexo masculino $(\mathrm{OR}=1,19)$, mais "novo" $(\mathrm{OR}=0,89)$ e despender mais tempo por semana em deslocamento ativo $(\mathrm{OR}=1,01)$ aumentam as chances de o adolescente realizar o deslocamento ativo em seu trajeto para a escola; enquanto que possuir carro em casa $(\mathrm{OR}=0,72)$ e perceber o trajeto para a escola como inseguro $(\mathrm{OR}=0,66)$ reduzem as chances de adoção a tal comportamento. Os resultados reforçam a característica multifatorial do deslocamento ativo, e a necessidade de intervenções centradas no sujeito e no ambiente.
\end{abstract}

Palavras-chave: Atividade física; Adolescência; Deslocamento ativo; Brasil.

\begin{abstract}
The purpose of this study is to describe the prevalence of active commuting among Brazilian adolescents in their route home-school by federal units and country regions, and to determine the predictors related to it. Sample comprised 99,967 adolescents (both sexes, mean age 14.24 years) that had taken part in the Brazilian National Adolescent School-based Health Survey in 2015. For the present study, it was considered information related to active commuting home-school (frequency and duration), socioeconomic status, and security perception at home-school route, which were obtained through questionnaire. Statistical analysis was performed by logistic regression. It was observed that $44.4 \%$ of adolescents reported to use the active commuting (go to and from school using bicycle or walking for, at least, five days a week), and the Southwest region showed the highest prevalence of adolescents who use the active commuting (46.4\%). Boys (OR = 1.19), younger adolescents $(O R=0.89)$, and those who spent more weekly time in active commuting $(O R$ $=1.01)$ are more prone to be adopt the active commuting in their route to and from school; on the other hand, having a car at home $(O R=0.72)$ and perceive the route tolfrom school as insecure $(O R=0.66)$ decrease their chances to adopt this behaviour. Results highlight that active commuting is a multifactorial trait, and the relevance to develop intervention strategies that should take into account both, subjects and the environment.
\end{abstract}

Keywords: Physical activity; Adolescence; Active commuting; Brazil.

\section{Introdução}

Os processos de urbanização e industrialização observados ao longo dos últimos anos trouxeram consigo alterações nos hábitos e estilo de vida da população ${ }^{1}$. Deste cenário, emergem as mudanças ocorridas na forma de deslocamento e envolvimento em atividade física (AF) habitual, o que tem levado ao aumento da inatividade física entre os jovens ${ }^{2}$.

Não obstante os efeitos deletérios da inatividade física, sabe-se que incrementos nos níveis de AF, mesmo de intensidade leve, podem atenuar esses problemas, com melhorias do perfil metabólico e redução do risco de morte prematura ${ }^{3}$. Nesse aspecto, o deslocamento ativo, caracterizado como sendo qualquer tipo de des- 
locamento em que o sujeito o faça de forma ativa, como por exemplo através de caminhada e/ou utilização de bicicleta ${ }^{4}$, tem sido apontado como um meio potencial de incrementos nos níveis de AF leve a moderada ${ }^{5}$, induzindo a melhoras dos aspectos de saúde 6 .

Especificamente para a população escolar, o deslocamento dos jovens no trajeto casa-escola pode ser visto como um momento relevante para promoção de estratégias visando o incremento de seus níveis de $\mathrm{AF}^{6}$. Contudo, observa-se uma baixa adesão ao uso dessa forma de deslocamento neste grupo ${ }^{7}$. Especificamente no contexto brasileiro, dados revelam que apenas cerca de $55 \%$ das crianças e jovens (6-21 anos) reportaram fazer uso do deslocamento ativo no trajeto casa-escola ${ }^{8}$. Tais informações ressaltam a necessidade de um mapeamento mais acurado com vistas à compreensão de como tal forma de AF se apresenta neste estrato etário. Adicionalmente, visto que apenas cerca de $31 \%$ dos jovens brasileiros são fisicamente ativos ${ }^{8}$, incrementos dos níveis totais de $\mathrm{AF}$, a partir do deslocamento ativo, pode ser uma estratégia relevante.

Portanto, compreender os fatores relacionados à adesão do deslocamento ativo pode fornecer informações que subsidiem o desenvolvimento de estratégias para seu incremento. Para isso, vale ressaltar a origem multifatorial da $\mathrm{AF}$, por forma que diversos fatores vão contribuir para sua expressão, dos quais pode-se ressaltar aspectos biológicos, demográficos, psicossociais, comportamentais, sociais, e ambientais ${ }^{9}$ e, especificamente no domínio do deslocamento, estes últimos (seja o ambiente natural, construído ou social) parecem exercer um papel singular em sua expressão ${ }^{10}$. Nesse sentido, considerar as diferenças socioculturais, econômicas e topográficas existentes entre os estados brasileiros ${ }^{11}$, torna-se relevante. Adicionalmente, considerando os baixos níveis de AF na população escolar ${ }^{8}$, bem como o fato de que hábitos adquiridos na infância tendem a perpetuar até a idade adulta ${ }^{12}$, torna-se relevante compreender o uso do deslocamento ativo por parte dos escolares brasileiros, bem como os fatores associados. Não obstante a relevância dos trabalhos prévios, e o significado de seus resultados, a maioria dos trabalhos conduzidos sob esta temática, com amostras brasileiras, envolveram adolescentes de um dado estado/região ${ }^{13}$, o que dificulta a generalização dos resultados, e não representa os escolares brasileiros em sua totalidade. Portanto, fornecer informações acerca do modo como os adolescentes brasileiros se deslocam no percurso casa-escola, bem como os preditores associados à adoção de tal comportamento, pode fornecer subsídios relevantes para o desenvolvimento de estratégias e políticas que visem promover incrementos na AF deste estrato etário. Dessa maneira, o presente estudo tem como objetivos i) descrever a frequência de deslocamento ativo no trajeto casa-escola entre adolescentes brasileiros, considerando as unidades federativas e regiões do país; e ii) determinar os preditores associados a tal comportamento entre adolescentes escolares.

\section{Métodos}

O presente estudo caracteriza-se por ser do tipo transversal, de base populacional, com análise secundária da Pesquisa Nacional de Saúde do Escolar (PeNSE), cuja coleta de informações ocorre a cada 3 anos, desde 2009, fruto de uma ação conjunta entre o Ministério da Saúde e o Instituto Brasileiro de Geografia e Estatística (IBGE), com apoio do Ministério da Educação (MEC). A pesquisa foi aprovada pela Comissão Nacional de Ética em Pesquisa (CONEP) (pareceres No 11.537/2009, No 16.805/2012, e No 1.006.467/2015), e informações acerca dos procedimentos de amostragem podem ser obtidas nos documentos previamente publicados $^{14}$.

Para o presente trabalho, a amostra foi composta por 99.967 adolescentes (51,3\% do sexo feminino), com média de idade de 14,24 anos (13 - 17 anos), distribuídos entre todas as unidades federativas do Brasil. O presente estudo foi conduzido com as informações referentes ao ano de 2015, envolvendo escolares do $9^{\circ}$ ano do Ensino Fundamental, regularmente matriculados em escolas públicas/privadas das capitais e interior das cinco regiões brasileiras ${ }^{14}$. A amostra considerada foi dimensionada com vistas a estimar parâmetros populacionais em diferentes domínios geográficos, a partir da estratificação geográfica do Território Nacional, em um total de 53 estratos, e tal estratificação foi considerada quando do procedimento de análise dos dados. Foram excluídos, para composição da amostra do presente estudo, participantes com idade inferior a 11 anos e superior a 17 anos, bem como os que apresentavam dados omissos para a variável dependente.

A coleta dos dados foi realizada entre abril e setembro de 2015, através do uso de questionário, aplicado via smartphones e distribuídos aos alunos presentes no dia das coletas das informações. Todos os alunos foram instruídos, por um técnico do IBGE, sobre os procedimentos para respostas ao questionário. $\mathrm{O}$ instrumento aplicado refere-se a um conjunto de informações re- 
lacionadas aos hábitos dos escolares. Para o presente trabalho, foram utilizadas informações acerca do deslocamento ativo para escola (fornecidas pelos estudantes, mediante resposta ao questionário, com base nos sete dias que antecederam à coleta de dados), bem como informações sociodemográficas, e informações passíveis de serem utilizadas enquanto indicador econômico e de segurança. Qualquer informação adicional relativamente aos aspectos metodológicos, podem ser consultados em relatório técnico publicado anteriormente ${ }^{14}$, a que acrescenta-se que o questionário utilizado apresentou validade satisfatória para estimativa da $\mathrm{AF}^{15}$. Os estudantes foram esclarecidos quanto aos procedimentos da pesquisa, sendo facultado o direito de não responder a qualquer questão e/ou deixar de participar da pesquisa a qualquer momento.

Informações sobre sexo e idade dos adolescentes foram indicadas durante a resposta ao questionário supracitado. Duas informações acerca do deslocamento ativo foram consideradas: frequência e tempo médio de deslocamento. Os adolescentes foram solicitados a indicar com que frequência realizam o percurso de ida e volta da escola através do uso de bicicleta/caminhada ("nenhum dia" a "5 dias mais sábado e domingo"), bem como o "tempo médio gasto" na realização de tal percurso (<10minutos, 10-19 minutos, 20-29 minutos, 30-9 minutos, 40-49 minutos, 50-59 minutos, $\geq 1$ hora). A partir das respostas à variável "frequência de deslocamento", os mesmos foram categorizados como a realizar "deslocamento ativo" (quando ida e volta da escola, em conjunto, foram realizadas através de bicicleta/caminhada em pelo menos 5 dias da semana), e como a realizar "deslocamento passivo" se tal forma de deslocamento deu-se em menos de 5 dias. A informação acerca do "tempo médio de deslocamento" corresponde ao tempo médio diário estimado acumulado pelos adolescentes (em minutos) em deslocamento escola-casa realizado a pé ou de bicicleta, considerando os últimos sete dias. Tal estimativa foi determinada a partir da multiplicação do número de dias reportados pelos adolescentes em que o deslocamento ativo foi realizado, pelo respectivo tempo gasto no deslocamento, fazendo-se uso do valor mediano do tempo (por exemplo, 15 minutos, se a resposta dada foi "10-19 minutos") [tempo médio de deslocamento $=$ (frequência de dias de deslocamento ativo na ida para a escola $\mathrm{X}$ tempo gasto na ida para escola) + (frequência de dias de deslocamento ativo na volta da escola $\mathrm{X}$ tempo gasto na volta da escola) $]^{16}$.
Considerando que a "presença de automóvel em casa" poderia condicionar a forma de deslocamento dos adolescentes, tal variável foi considerada com um indicador socioeconômico. A variável foi dicotomizada em "possui" ou "não possui" carro para locomoção.

Para determinar a percepção de segurança dos adolescentes, fez-se uso de informações acerca do número de vezes, nos últimos 30 dias, que os estudantes reportaram não ter comparecido às aulas por não se sentir seguro no trajeto realizado para a escola. Dessa forma, a variável foi dicotomizada em "nenhuma falta" e " $\geq 1$ falta".

As informações descritivas foram apresentadas a partir de média (erro padrão) e/ou frequências (\%) (IC95\%), estratificados por unidades federativas. Para verificar a existência de diferenças significativas na frequência de adolescentes que realizam deslocamento ativo, entre as regiões brasileiras, foi utilizado o teste de qui-quadrado.

A associação entre os preditores e a variável dependente foi estimada através da análise de regressão logística binária, por regiões e posteriormente considerando a amostra total. A variável dependente foi dicotomizada em "deslocamento passivo" e "deslocamento ativo", tendo como preditores características do indivíduo - sexo, idade, tempo médio de deslocamento/semana; fatores econômicos - presença de automóvel em casa; e características do ambiente - percepção de segurança no caminho para a escola, sendo inseridos na análise apenas os sujeitos com informações válidas para todas as variáveis consideradas $(\mathrm{n}=99.276)$. Com o intuito de verificar os preditores de adoção de deslocamento ativo, foram construídos modelos brutos (para cada uma das variáveis independentes) e o modelo final, ajustado a partir da inserção de todas as variáveis preditoras. Todas as análises foram realizadas no software SPSS 24.0, sendo considerado o valor de $\mathrm{p}<0,05$. Em todas as análises, foi utilizado procedimento de ponderação, conforme instruções apresentadas no protocolo para análise de dados da PeNSE 2015.

\section{Resultados}

Informações da análise descritiva indicam que a amostra apresenta média de idade de 14,24 $(0,01)$ anos, e grande variação interestadual acerca dos valores médios de tempo de deslocamento, com os menores valores observados para o estado do Piauí $[66,94(3,27)$ minutos], contrapondo-se ao observado no estado de Pernambuco $[107,07(4,68)$ minutos]. Os jovens dos estados de Santa Catarina e Sergipe foram os que menos repor- 
taram faltar à escola em decorrência do sentimento de insegurança ao longo do trajeto, contrapondo-se ao reportado pelos adolescentes do estado do Mato Grosso, onde $15,3 \%$ dos participantes relataram ter faltado à escola pelo menos uma vez nos últimos 30 dias. A região Norte do Brasil apresentou menor frequência de existência de carro na residência, enquanto a maior frequência foi observada na região Sul (Tabela 1).
A análise sobre o deslocamento ativo dos adolescentes brasileiros evidencia dois pontos interessantes: 1) a nível nacional, $44,4 \%$ dos adolescentes realizam o deslocamento ativo durante o trajeto para a escola, e 2) existem diferenças significativas na frequência de deslocamento ativo entre as cinco regiões do país ( $\mathrm{p}=$ 0.005), com a região Sudeste apresentando as maiores percentagens $(46,4 \%)$, e os valores mais baixos sendo

Tabela 1 - Informação descritiva das variáveis relacionadas ao deslocamento ativo (tempo médio e classificação), indicador econômico e percepção de seguraça dos adolescentes brasileiros (estimativas ponderadas, respeitando-se o peso amostral).

\begin{tabular}{|c|c|c|c|c|c|c|c|}
\hline \multirow{2}{*}{ Estados } & \multirow{2}{*}{$\begin{array}{c}\text { Tempo médio } \\
\text { deslocamento } \\
\text { semanal (min) } \\
(\mathrm{n}=99.934) \\
\text { Média } \\
\text { (Erro Padrão) }\end{array}$} & \multicolumn{2}{|c|}{$\begin{array}{c}\text { Deslocamento } \\
(\mathrm{n}=99.967)\end{array}$} & \multicolumn{2}{|c|}{$\begin{array}{l}\text { Possui carro } \\
(\mathrm{n}=99.894)\end{array}$} & \multicolumn{2}{|c|}{$\begin{array}{l}\text { Faltou à escola (insegurança) } \\
\qquad(\mathrm{n}=99.369)\end{array}$} \\
\hline & & $\begin{array}{l}\text { Passivo\% } \\
\text { (IC95\%) }\end{array}$ & $\begin{array}{c}\text { Ativo\% } \\
\text { (IC95\%) }\end{array}$ & $\begin{array}{c}\text { Não\% } \\
(\mathrm{IC} 95 \%)\end{array}$ & $\begin{array}{c}\mathrm{Sim} \% \\
(\mathrm{IC} 95 \%)\end{array}$ & $\begin{array}{l}\text { Nenhum dia\% } \\
\text { (IC95\%) }\end{array}$ & $\begin{array}{l}\geq 1 \mathrm{dia} \% \\
(\mathrm{IC} 95 \%)\end{array}$ \\
\hline Rondônia & $91,36(4,62)$ & $54,4(49,9-58,8)$ & $45,6(41,2-50,1)$ & $46,5(42,7-50,3)$ & $53,5(49,7-57,3)$ & $92,0(90,7-93,3)$ & $8,0(6,7-9,3)$ \\
\hline Acre & $105,84(4,43)$ & $56,9(52,8-60,9)$ & $43,1(39,1-47,2)$ & $66,0(62,0-69,8)$ & $34,0(30,2-38,0)$ & $91,9(90,7-92,9)$ & $8,1(7,1-9,3)$ \\
\hline Amazonas & $91,86(4,31)$ & $58,6(54,7-62,5)$ & $41,4(37,5-45,3)$ & $68,0(64,2-71,6)$ & $32,0(28,4-35,8)$ & $86,7(84,9-88,3)$ & $13,3(11,7-15,1)$ \\
\hline Roraima & $86,20(5,34)$ & $61,9(57,3-66,3)$ & $38,1(33,7-42,7)$ & $51,7(47,4-56,0)$ & $48,3(44,0-52,6)$ & $88,4(87,0-89,7)$ & $11,6(10,3-13,0)$ \\
\hline Pará & $87,71(3,48)$ & $55,1(50,5-59,6)$ & $44,9(40,4-49,5)$ & $68,3(65,0-71,5)$ & $31,7(28,5-35,0)$ & $88,3(86,5-89,9)$ & $11,7(10,1-13,5)$ \\
\hline Amapá & $91,68(3,81)$ & $56,1(52,4-59,8)$ & $43,9(40,2-47,6)$ & $60,8(57,0-64,4)$ & $39,2(35,6-42,9)$ & $87,0(85,8-88,0)$ & $13,0(12,0-14,2)$ \\
\hline Tocantins & $88,07(5,07)$ & $53,9(50,3-57,5)$ & $46,1(42,5-49,7)$ & $49,8(45,9-53,6)$ & $50,2(46,4-54,1)$ & $89,6(87,5-91,4)$ & $10,4(8,6-12,5)$ \\
\hline Maranhão & $79,15(3,13)$ & $60,2(57,2-63,2)$ & $39,8(36,8-42,8)$ & $70,5(67,8-73,1)$ & $29,5(26,9-32,2)$ & $89,9(88,4-91,1)$ & $10,1(8,9-11,6)$ \\
\hline Piauí & $66,94(3,27)$ & $63,5(59,9-67,0)$ & $36,5(33,0-40,1)$ & $67,4(64,6-70,1)$ & $32,6(29,9-35,4)$ & $91,9(90,7-93,0)$ & $8,1(7,0-9,3)$ \\
\hline Ceará & $78,11(3,65)$ & $61,3(57,8-64,8)$ & $38,7(35,2-42,2)$ & $68,2(65,8-70,5)$ & $31,8(29,5-34,2)$ & $88,7(87,3-89,9)$ & $11,3(10,1-12,7)$ \\
\hline $\begin{array}{l}\text { Rio Grande } \\
\text { do Norte }\end{array}$ & $76,55(3,41)$ & $61,0(57,4-64,5)$ & $39,0(35,5-42,6)$ & $54,8(52,0-57,6)$ & $45,2(42,4-48,0)$ & $87,9(86,2-89,3)$ & $12,1(10,7-13,8)$ \\
\hline Paraíba & $78,75(4,18)$ & $59,9(55,4-64,1)$ & $40,1(35,9-44,6)$ & $55,6(53,2-58,0)$ & $44,4(42,0-46,8)$ & $87,8(85,9-89,4)$ & $12,2(10,6-14,1)$ \\
\hline Pernambuco & $107,07(4,68)$ & $49,8(45,9-53,6)$ & $50,2(46,4-54,1)$ & $60,9(58,0-63,7)$ & $39,1(36,3-42,0)$ & $85,6(84,1-87,0)$ & $14,4(13,0-15,9)$ \\
\hline Alagoas & $82,68(5,41)$ & $58,2(51,9-64,3)$ & $41,8(35,7-48,1)$ & $63,1(59,2-66,8)$ & $36,9(33,2-40,8)$ & $88,7(87,2-90,0)$ & $11,3(10,0-12,8)$ \\
\hline Sergipe & $67,12(3,00)$ & $61,4(58,0-64,6)$ & $38,6(35,4-42,0)$ & $58,8(56,1-61,4)$ & $41,2(38,6-43,9)$ & $92,4(91,2-93,3)$ & $7,6(6,7-8,8)$ \\
\hline Bahia & $88,46(5,36)$ & $59,2(53,9-64,2)$ & $40,8(35,8-46,1)$ & $59,6(56,4-62,5)$ & $40,5(37,5-43,6)$ & $88,6(86,7-90,2)$ & $11,4(9,8-13,3)$ \\
\hline Minas Gerais & $94,98(3,85)$ & $48,6(44,6-52,7)$ & $51,4(47,3-55,4)$ & $36,6(33,4-40,0)$ & $63,4(60,0-66,6)$ & $90,0(88,6-91,3)$ & $10,0(8,7-11,4)$ \\
\hline $\begin{array}{l}\text { Espírito } \\
\text { Santo }\end{array}$ & $84,67(3,50)$ & $45,0(40,8-49,2)$ & $55,0(50,8-59,2)$ & $43,2(40,8-45,7)$ & $56,8(54,3-59,2)$ & $90,0(88,5-91,3)$ & $10,0(8,7-11,5)$ \\
\hline Rio de Janeiro & $93,27(3,47)$ & $53,1(49,4-56,7)$ & $46,9(43,3-50,6)$ & $43,0(40,4-45,6)$ & $57,0(54,4-59,6)$ & $85,9(84,2-87,5)$ & $14,1(12,5-15,8)$ \\
\hline São Paulo & $87,84(4,68)$ & $56,9(52,8-60,9)$ & $43,1(39,1-47,2)$ & $29,2(26,7-31,9)$ & $70,8(68,1-73,3)$ & $87,4(85,3-89,2)$ & $12,6(10,8-14,7)$ \\
\hline Paraná & $86,78(4,15)$ & $55,6(50,8-60,3)$ & $44,4(39,7-49,2)$ & $20,2(18,2-22,4)$ & $79,8(77,6-81,8)$ & $92,1(90,8-93,2)$ & $7,9(6,8-9,2)$ \\
\hline $\begin{array}{l}\text { Santa } \\
\text { Catarina }\end{array}$ & $77,35(4,21)$ & $58,2(53,5-62,7)$ & $41,8(37,3-46,5)$ & $19,7(17,7-22,0)$ & $80,3(78,0-82,3)$ & $93,8(92,7-94,7)$ & $6,2(5,3-7,3)$ \\
\hline $\begin{array}{l}\text { Rio Grande } \\
\text { do sul }\end{array}$ & $76,16(5,06)$ & $51,8(46,2-57,4)$ & $48,2(42,6-53,8)$ & $28,2(25,1-31,4)$ & $71,8(68,6-74,9)$ & $90,5(88,5-92,2)$ & $9,5(7,8-11,5)$ \\
\hline $\begin{array}{l}\text { Mato Grosso } \\
\text { do Sul }\end{array}$ & $85,49(3,43)$ & $56,2(52,0-60,3)$ & $43,8(39,7-48,0)$ & $34,8(31,7-38,2)$ & $65,2(61,8-68,3)$ & $88,7(85,6-91,2)$ & $11,3(8,8-14,4)$ \\
\hline Mato Grosso & $95,47(4,44)$ & $56,8(52,6-60,8)$ & $43,2(39,2-47,4)$ & $35,1(31,6-38,8)$ & $64,9(61,2-68,4)$ & $84,7(82,1-86,9)$ & $15,3(13,1-17,9)$ \\
\hline Goiás & $98,05(3,30)$ & $54,2(51,1-57,3)$ & $45,8(42,7-48,9)$ & $32,7(30,6-34,8)$ & $67,3(65,2-69,4)$ & $89,1(87,9-90,2)$ & $10,9(9,8-12,1)$ \\
\hline $\begin{array}{l}\text { Distrito } \\
\text { Federal }\end{array}$ & $79,05(4,87)$ & $58,8(51,8-65,4)$ & $41,2(34,6-48,2)$ & $23,4(18,8-28,7)$ & $76,6(71,3-81,2)$ & $88,0(85,3-90,4)$ & $12,0(9,6-14,7)$ \\
\hline
\end{tabular}

IC95\% = Intervalo de confiança 95\% 
observados na região Nordeste (41,4\%) (Figura 1). Quando os resultados são apresentados por unidades federativas, verifica-se uma variabilidade intra-regional, sendo as unidades federativas em que os adolescentes mais realizam o deslocamento ativo, por região: Tocantins (região Norte - 46,1\%), Pernambuco (região Nordeste - 50,2\%), Espírito Santo (região Sudeste - 55,0\%), Rio Grande do Sul (região Sul - 48,2\%) e Goiás (região Centro-oeste-45,8\%).

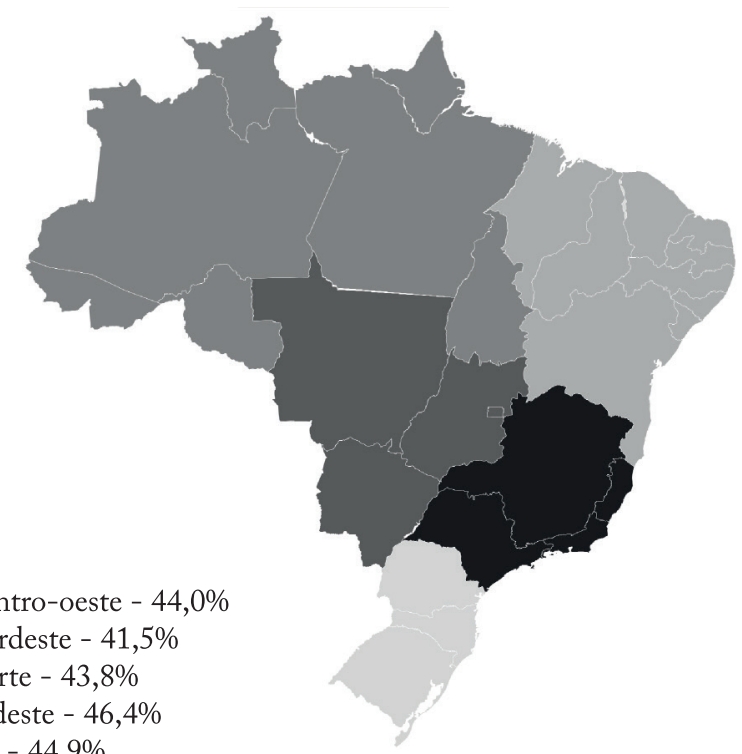

- Sudeste - 46,4\% Sul - 44,9\%

Powered by Bing @ Geoframes, Microsoft, Tom Tom

Figura 1 - Deslocamento ativo consoante as cinco regiões do Brasil.

Resultados da regressão logística, acerca dos preditores do deslocamento ativo dos jovens, estão apresentados na Tabela 2. Visto que os resultados da análise por regiões não diferiram em geral, em termos de significância dos preditores, mostrando o mesmo padrão dos resultados observados quando considerada a amostra total, a Tabela 2 apenas apresenta os resultados sem estratificação por regiões. Das variáveis inerentes ao sujeito, observa-se que as variáveis sexo, idade e tempo médio de deslocamento/semana apresentaram-se como preditores significativos para o deslocamento ativo dos adolescentes, indicando que ser do sexo masculino (OR = 1,19; IC95\%: $1,13-1,25)$, ser mais "novo" $(\mathrm{OR}=$ 0,89; IC95\%: 0,85-0,93) e despender mais tempo por semana em deslocamento ativo $(\mathrm{OR}=1,01$; IC $95 \%$ : 1,01 - 1,02) aumentam as chances de o adolescente realizar o deslocamento ativo na ida e volta para/da escola em, pelo menos, 5 dias da semana. Por outro lado, possuir carro em casa $(\mathrm{OR}=0,72$; IC95\%: 0,67 - 0,77) e perceber o trajeto para a escola como inseguro (OR = 0,66; IC95\%: 0,60-0,73) reduzem as chances de o adolescente ser ativo em seu deslocamento.

\section{Discussão}

A proposta do presente trabalho foi descrever a frequência de uso do deslocamento ativo no trajeto casa-escola entre adolescentes brasileiros, considerando as unidades federativas e regiões, bem como determinar os preditores associados a tal comportamento. Dada a grande extensão territorial e diferenças entre as cinco regiões do país, a verificação da frequência de adoção desse comportamento entre os adolescentes foi feita a partir de três vertentes: 1) considerando todo o Brasil, 2) consoante regiões geográficas e 3) considerando as unidades federativas do país. Considerando a amostra total, $44,4 \%$ dos adolescentes brasileiros realizam o deslocamento de forma ativa em pelo menos cinco dias durante a semana. Informações oriundas de diferentes regiões brasileiras, mostram-se díspares quanto ao uso do deslocamento ativo por parte dos escolares no trajeto casa-escola. Por exemplo, Silva et al. ${ }^{17}$, estudando jovens da cidade de João Pessoa (Paraíba), reportaram que $70,4 \%$ dos jovens amostrados faziam uso do deslocamento ativo para a escola, similarmente ao encontrado entre adolescentes da cidade de Uruguaiana (Rio Grande do Sul), onde tal comportamento foi observado em $75,7 \%$ da amostra estudada ${ }^{18}$. Em um estudo de tendência decenal conduzido na cidade de São Paulo, entre os anos de 1997-2007, Sá et al. ${ }^{19}$. reportaram uma ligeira redução na adoção deste com-

Tabela 2 - Resultados da regressão logística para os preditores do deslocamento ativo dos adolescentes.

\begin{tabular}{|c|c|c|c|c|c|c|}
\hline \multirow{2}{*}{ Variáveis inseridas no modelo } & \multicolumn{3}{|c|}{ Modelo bruto } & \multicolumn{3}{|c|}{ Modelo ajustado } \\
\hline & OR & $\mathrm{p}$ & $\mathrm{IC} 95 \%$ & OR & $\mathrm{p}$ & $\mathrm{IC} 95 \%$ \\
\hline Sexo (masculino) & 1,10 & $<0,001$ & $1,05-1,15$ & 1,19 & $<0,001$ & $1,13-1,25$ \\
\hline Idade (anos) & 0,97 & 0,047 & $0,93-0,99$ & 0,89 & $<0,001$ & $0,85-0,93$ \\
\hline Possuir carro (sim) & 0,67 & $<0,001$ & $0,62-0,71$ & 0,72 & $<0,001$ & $0,67-0,77$ \\
\hline Percepção de Segurança no caminho para a escola ( $\geq 1$ falta) & 0,95 & 0,154 & $0,88-1,02$ & 0,66 & $<0,001$ & $0,60-0,73$ \\
\hline Tempo médio de deslocamento semanal (min) & 1,02 & $<0,001$ & $1,014-1,015$ & 1,01 & $<0,001$ & $1,01-1,02$ \\
\hline
\end{tabular}

IC95\% = Intervalo de confiança 95\% 
portamento entre os rapazes, ao passo que não foram observadas alterações entre as meninas, situando-se os valores de frequência próximo ao encontrado nos trabalhos citados anteriormente (de $71 \%$ para $67 \%$ entre os meninos; e de $68 \%$ para $69 \%$ entre as meninas). Por outro lado, menos de $21 \%$ dos adolescentes escolares de Pernambuco reportaram ser fisicamente ativos no deslocamento para a escola ${ }^{20}$. A nível mundial, tal disparidade também é passível de ser observada. Embora não haja dados que reportem a prevalência mundial do uso do deslocamento ativo por parte de adolescentes, trabalhos de revisão, que objetivaram associar o deslocamento ativo a outra variável/comportamento, encontraram prevalências de deslocamento ativo oscilando entre $21,4 \%$ e $86,4 \%$ (para adolescentes portugueses e dinamarqueses, respectivamente) ${ }^{21}$, e entre $23 \%$ e 67,0\% (para adolescentes chilenos e adolescentes espanholas $)^{22}$. No Brasil, embora seja evidenciado um aumento de aproximadamente $21 \%$ na malha cicloviária das capitais do país, menos de $6 \%$ das cidades brasileiras possuem plano de mobilidade, dificultando a utilização da bicicleta como um meio de transporte ${ }^{23}$.

Levando em consideração a análise estratificada por regiões, a frequência de deslocamento ativo apresentou-se inferior a 50\% em todas as regiões, sendo a região Sudeste aquela que apresentou a maior porcentagem $(46,4 \%)$. Tais resultados tornam-se interessantes quando da perspectiva de que mesmo existindo grande variabilidade em relação aos fatores climáticos, econômicos e culturais entre as regiões, que podem responder por diferenças no uso do transporte ativo por parte da população ${ }^{24}$, essas diferenças não refletiram no objeto de estudo do presente trabalho. Além da verificação de frequências, a análise de regressão logística também foi conduzida por regiões, onde observou-se, na generalidade, uma similaridade no que diz respeito aos preditores associados ao comportamento supracitado (entre as regiões, bem como comparativamente aos resultados considerando a amostra total), motivo pelo qual optou-se pela apresentação dos resultados dos preditores sem estratificar a amostra por regiões (a que se associa a perspectiva de internacionalização da informação, visto ser mais facilmente compreendida e comparável entre pesquisadores internacionais).

A verificação da frequência de deslocamento ativo entre as unidades federativas indica que apenas em Pernambuco (50,2\%), Minas Gerais $(51,4 \%)$ e no Espírito Santo $(55,0 \%)$ mais da metade dos escolares foram classificados como fisicamente ativos em seu desloca- mento para a escola. Tais resultados, quando confrontados com trabalhos anteriores, sublinham as diferenças entre os estados brasileiros no tocante ao percentual de adolescentes que se deslocam ativamente. Em uma revisão de literatura conduzida por Ferrari et al. ${ }^{13}$ acerca do deslocamento ativo para a escola por parte dos escolares brasileiros, foram sumariados valores de adesão a tal comportamento inferiores a $60 \%$ na maior parte dos estudos, com variações entre $34,3 \%$ e $75,7 \%$.

Os fatores associados a tais diferenças são de natureza multivariada, e podem ser influenciados tanto por características do sujeito (como sexo e condição econômica), quanto do ambiente no qual o mesmo está inserido. Daqui que, no presente estudo, rapazes apresentaram maior propensão a serem ativos no deslocamento, fato esse que pode estar associado a maior preocupação com aspectos de segurança no público feminino (seja pela percepção própria do grupo, como também pelo cuidado/preocupação dos pais que tendem a proteger mais as filhas, comparativamente aos filhos ${ }^{25}$, bem como estereótipos culturais que tendem a estimular adoção de comportamentos menos ativos entre as meninas. Além disso, não obstante as intempéries do ambiente natural e condições climáticas estarem associadas à adesão do comportamento em ambos os sexos ${ }^{10}$, é possível que preocupações das meninas quanto a aspectos relacionados à sua aparência frente a seus pares, que pode ser afetada por tais fatores (transpiração, alterações de penteado, uso de roupas adequadas/confortáveis), seja uma condicionante extra a um menor uso pelas mesmas da forma ativa de deslocar-se para a escola.

Concomitantemente, estudos prévios têm reportado a existência de uma relação entre $\mathrm{AF}$ e percepção do ambiente físico com o sexo, demonstrando que para os meninos as características do ambiente isolado (existência de calçadas e ciclovias) são fatores associados à $\mathrm{AF}$ global, o que não ocorre no sexo feminino ${ }^{26}$. Além disso, estudos relatam que a preocupação com a segurança pessoal e no trânsito, bem como a existência de locais disponíveis para prática do deslocamento ativo (passarelas, calçadas e ciclovias) e a distância casa-escola, são fatores importantes para a adoção de comportamentos ativos ${ }^{10}$.

Acrescenta-se, ainda, a importância de se considerar o fator "econômico" e "idade" no referido comportamento, sobretudo a partir da percepção de distância do percurso casa-escola, pois tal fato tende a ser amenizado pela utilização de transporte privado, principalmente no público mais novo, visto que este grupo é mais dependente de seus responsáveis, o que leva a um menor 
tempo diário despendido em deslocamento ativo. Esta ideia fora previamente reportada por Sá et al. ${ }^{27}$, através da Pesquisa de mobilidade, realizada em 2012, em que foi verificada uma celeridade e continuidade na queda do uso de transporte ativo entre crianças no estado de São Paulo (entre 1997-2012), devido ao incremento no uso de meios de transporte motorizado privado (passando de 23,7\% em 1997, para 46,9\% em 2012).

Tendo em vista a possibilidade de manutenção de hábitos adquiridos na infância durante a adolescência e vida adulta, e observando os baixos níveis de deslocamento ativo evidenciado pelo estudo, tais resultados associam-se e alertam sobre a necessidade do entendimento de fatores que levam à adoção desse comportamento em tenra idade, bem como a perspectiva do que denomina-se marginal gains, ou seja, pequenas mudanças realizadas em diferentes aspectos, quando somadas em uma perspectiva a longo prazo, conduzem a ganhos substanciais $^{28}$. Trazendo esta ideia para o contexto da $\mathrm{AF}$, percebe-se que incrementos na $\mathrm{AF}$ no domínio do deslocamento podem induzir a aumentos na AF global do sujeito e, neste contexto, a realização do deslocamento ativo durante o trajeto para a escola pode conduzir a benefícios para a saúde, dado ser uma prática realizada diariamente pela população escolar ${ }^{6}$.

Das limitações do presente trabalho pode-se referir 1) o uso de informação auto reportada acerca da AF dos sujeitos, o que pode estar susceptível a erros; contudo, outros estudos no domínio da epidemiologia da AF têm sido conduzidos com estratégias semelhantes no tocante a instrumentos para coleta de dados; e 2) a ausência de outras variáveis acerca do ambiente, que podem estar associadas à adoção do deslocamento ativo por parte dos adolescentes. Apesar das limitações, alguns pontos positivos merecem ser destacados, tais como a utilização de uma amostra representativa dos escolares do $9^{\circ}$ ano do Ensino Fundamental do país e o uso de preditores individuais e ambientais que podem estar associados ao envolvimento de adolescentes na realização de deslocamento ativo.

Embora não tenha sido objeto de estudo do presente trabalho, é possível especular que o design urbano das cidades também pode ser considerado um fator decisório quanto ao uso do deslocamento ativo para ir/voltar da escola por parte dos adolescentes ${ }^{29}$. A reorganização das cidades brasileiras, para que se tornem mais compactas e acessíveis ao uso de formas ativas de deslocamento, para além de políticas públicas que conduzam à existência de ambientes mais seguros, podem favorecer o uso desta forma de deslocamento por parte da populaçãa ${ }^{10}$.

Nesse sentido, o presente trabalho indica que menos da metade dos adolescentes brasileiros (44\%) realizam deslocamento ativo no trajeto para a escola. Dos fatores associados a esse comportamento destacam a influência de características biológicas (sexo e idade), comportamentais (tempo médio de deslocamento), ambientais (percepção de segurança durante o trajeto) e econômicas (possuir automóvel em casa), reafirmando o caráter multifatorial desse domínio da $\mathrm{AF}$ e a necessidade de intervenções centradas no sujeito e no ambiente. Estas informações podem servir de subsídios para planejamento e desenvolvimento de estratégias com fins à promoção da atividade física de escolares, a partir do uso do deslocamento ativo.

\section{Conflito de interesse}

Os autores declaram não haver conflito de interesse.

\section{Contribuição dos autores}

Thuany M, participou da concepção inicial do estudo, produção e revisão do manuscrito. Santos FK, participou da revisão crítica do manuscrito. Almeida $\mathrm{MB}$, participou da revisão crítica do manuscrito. Gomes TNQF, participou da concepção inicial do estudo, produção do manuscrito e revisão crítica do texto. Todos os autores concordaram com a redação final do trabalho.

\section{Referências}

1. Katzmarzyk PT, Mason C. The physical activity transition. J Phys Act Health. 2009;6(3):269-80.

2. Guthold R, Stevens GA, Riley LM, Bull FC. Global trends in insufficient physical activity among adolescents: a pooled analysis of 298 population-based surveys with 1.6 million participants. Lancet Child Adolesc Health. 2020;4(1):23-35.

3. Poitras VJ, Gray CE, Borghese MM, Carson V, Chaput J-P, Janssen I, et al. Systematic review of the relationships between objectively measured physical activity and health indicators in school-aged children and youth. Appl Physiol Nutr Metab. 2016;41(6 Suppl 3):S197-239.

4. Mitra R, Buliung RN. Built environment correlates of active school transportation: neighborhood and the modifiable areal unit problem. J Transp Geogr. 2012;20(1):51-61.

5. Burgos MS, Tornquist D, Tornquist L, Reuter CP, Garcia EL, Renner JDP, et al. Cardiometabolic risk factors associated with active commuting to school. Rev Paul Pediatr. 2019;37(2):181-7.

6. Larouche R, Saunders TJ, Faulkner G, Colley R, Tremblay M. Associations between active school transport and physical activity, body composition, and cardiovascular fitness: a systematic review of 68 studies. J Phys Act Health. 2014;11(1):206-27.

7. Aubert S, Barnes JD, Abdeta C, Nader PA, Adeniyi AF, Aguilar-Farias N, et al. Global Matrix 3.0 Physical Activity Report Card Grades for children and youth: Results and analysis from 49 countries. J Phys Act Health. 2018;15(S2):S251-S73. 
8. Silva DAS, Christofaro DGD, Ferrari GLM, Silva KS, Nardo N, Silva RJS, et al. Results from Brazil's 2018 Report Card on physical activity for children and youth. J Phys Act Health. 2018;15(S2):S323-S5.

9. Bauman AE, Reis RS, Sallis JF, Wells JC, Loos RJF, Martin BW. Correlates of physical activity: why are some people physically active and others not? Lancet. 2012;380(9838):258-71.

10. Rahman ML, Moore A, Smith M, Lieswyn J, Mandic S. A conceptual framework for modelling safe walking and cycling routes to high schools. Int J Environ Res Public Health. 2020;17(9).

11. Programa das Naçõe Unidas para o Desenvolvimento (PNUD), Instituto de Pesquisa Econômica Aplicada (IPEA), Fundação João Pinheiro (FJP). Atlas do Desenvolvimento Humano no Brasil, 2014. [citado em 2020 jan]. Disponível em: http://atlasbrasil.org.br/2013/pt/home/.

12. Rovio SP, Yang X, Kankaanpää A, Aalto V, Hirvensalo M, Telama R, et al.Longitudinal physical activity trajectories from childhood to adulthood and their determinants: The Young Finns Study. Scand J Med Sci Sports. 2018;28(3):1073-83.

13. Ferrari GLM, Victo ER, Ferrari TK, Solé D. Active transportation to school for children and adolescents from Brazil: a systematic review. Rev Bras Cineantropom Desempenho Hum. 2018;20(4):406-14.

14. Instituto Brasileiro de Geografia e Estatística (IBGE). Pesquisa Nacional de Saúde do Escolar (PeNSE) 2015. Rio de Janeiro: IBGE, 2016.

15. Tavares LF, Castro IR, Cardoso LO, Levy RB, Claro RM, Oliveira AF. Validade de indicadores de atividade fisica e comportamento sedentario da Pesquisa Nacional de Saude do Escolar entre adolescentes do Rio de Janeiro, Brasil. Cad. de Saúde Pública. 2014;30(9):1861-74.

16. Instituto Brasileiro de Geografia e Estatística (IBGE). Pesquisa Nacional de Saúde do Escolar 2015. Nota metodológica no 2 - Especificações de tableas e indicadores Amostra 1. Brasil, grandes regioões, unidades da federação e municípios das capitais. Rio de Janeiro: IBGE; 2018.

17. Silva KS, Lopes AS, Silva FM. Atividade física no deslocamento à escola e no tempo livre em crianças e adolescentes da cidade de João Pessoa, PB, Brasil. R Bras Ci e Mov. 2007;15(3):61-70.

18. Streb A, Graup S, Bergmann M, Bergmann G. Excesso de peso e deslocamento para a escola em adolescentes de Uruguaiana/RS. Rev Bras Ativ Fís Saúde. 2016;21(3):255-62.
19. Sá TH, Garcia LMT, Mielke GI, Rabacow FM, Rezende LFM. Changes in travel to school patterns among children and adolescents in the São Paulo Metropolitan Area, Brazil, 1997-2007. J Transp Health. 2015;2(2):143-50.

20. Santana FS, Palmeira AC, Santos MAM, Farah BQ, Souza BCC, Ritti-Dias RM. Associação entre deslocamento ativo e pressão arterial elevada em adolescentes. Einstein. 2017;15(4):415-20.

21. Pinto AA, Claumann GS, Angelo HCC, Menezes EC, Dias DT, Pelegrini A. Active commuting to school and associated factors among adolescents: A systematic review. J Phys Educ. 2017;28:e2859.

22. Phansikar M, Ashrafi SA, Khan NA, Massey WV, Mullen SP. Active commute in relation to cognition and academic achievement in children and adolescents: A systematic review and future recommendations. Int J Environ Res Public Health. 2019;16(24).

23. Associação Abaporu. Mobilize - mobilidade urbana sustentável, 2019. [citado em 2019 dez]. Disponível em: https://www.mobilize.org.br/.

24. Sá TH, Pereira RHM, Duran AC, Monteiro CA. Socioeconomic and regional differences in active transportation in Brazil. Rev Saude Publica. 2016; 50.

25. Christian HE, Klinker CD, Villanueva K, Knuiman MW, Foster SA, Zubrick SR, et al. The effect of the social and physical environment on children's independent mobility to neighborhood destinations.J Phys Act Health.2015;12(Suppl 1):S84 -S93.

26. Lopes AAS, Lanzoni AN, Hino AAF, Rodriguez-Añez CR, ReisI RS. Perceived neighborhood environment and physical activity among high school students from Curitiba, Brazil. Rev Bras Epidemiol. 2014;17(4):938-53.

27. Sá TH, Rezende LFM, Rabacow FM, Monteiro CA. Use of private motor vehicle transportation for taking children to school in Sao Paulo Metropolitan Area, Brazil, 1997-2012. Cad. de Saúde Pública. 2016;32(5):e00140215.

28. Smith WB. Marginal Gains. Foot Ankle Spec. 2018; 11(4):306-7.

29. Kleinert $S$, Horton R. Urban design: an important future force for health and wellbeing. Lancet. 2016; 388(10062):2848-50.

Recebido: 27/08/2020

Aprovado: 24/05/2021 